\title{
Endoscopic submucosal dissection of gastric subepithelial tumors: a systematic review and meta-analysis
}

\author{
Chang Seok Bang ${ }^{1}$, Gwang Ho Baik ${ }^{1}$, In Soo Shin ${ }^{2}$, Ki Tae Suk ${ }^{1}$, Jai Hoon Yoon ${ }^{1}$, and Dong Joon Kim ${ }^{1}$
}

\author{
${ }^{1}$ Department of Internal Medicine, \\ Hallym University College of \\ Medicine, Chuncheon; ${ }^{2}$ Jeonju \\ University College of Education, \\ Jeonju, Korea
}

Background/Aims: To evaluate the therapeutic outcomes of the endoscopic submucosal dissection (ESD) technique for the treatment of gastric subepithelial tumors (SETs).

Methods: A systematic literature review was conducted using the core databases. Data on the complete resection rates and the procedure-related perforation rates were extracted and analyzed. A random effects model was then applied for this meta-analysis.

Results: In all, 288 patients with 290 SETs were enrolled from nine studies (44 SETs originated from the submucosal layer; 246 SETs originated from the muscularis propria layer). The mean diameter of the lesions ranged from 17.99 to 38 $\mathrm{mm}$. Overall, the pooled complete resection rate was estimated to be $86.2 \%$ (95\% confidence interval [CI], 78.9 to 91.3). If the analysis was limited to the lesions that originated from the submucosal layer, the pooled complete resection rate was 91.4\% (95\% CI, 77.9 to 97). If the analysis was limited to the lesions that originated from the muscularis propria, the pooled complete resection rate was $84.4 \%$ (95\% CI, 78.7 to 88.8 ). The pooled procedure-related gastric perforation rate was $13 \%$ (95\% CI, 9.4 to 17.6). Sensitivity analyses showed consistent results. Finally, publication bias was not detected.

Conclusions: ESD, including endoscopic muscularis dissection, is a technically feasible procedure for the treatment of SETs. However, selection bias is suspected from the enrolled studies. For the development of a proper indication of ESD for SETs, further studies are needed.

Keywords: Endoscopic submucosal dissection; Gastrointestinal stromal tumors; Subepithelial tumors

\section{INTRODUCTION}

Gastric subepithelial tumors (SETs) are commonly encountered during upper endoscopic examination, and with the widespread use of screening endoscopy, the detection rate of SETs has continued to increase. Large SETs and symptomatic lesions that require surgical treat- ment usually do not require a pre-operative tissue diagnosis [1]. Small SETs, especially lesions less than $1 \mathrm{~cm}$ in size, are recommended for regular endoscopic follow-up without tissue diagnosis or treatment [2]. However, intermediate-sized lesions require a differential diagnosis due to the difference in therapeutic approaches between SETs with and without malignant potential. Tissue diag- 
nosis is difficult because SETs are covered with normal epithelium, and the layer of origin cannot be assessed by conventional endoscopy. Periodic endoscopy with endoscopic ultrasound (EUS) is usually performed for this type of lesion in order to characterize and identify SETs; in addition, the hypoechoic masses that may originate from the 3 rd or 4 th echo layer require histological confirmation [3]. However, no consensus exists as to the use of endoscopic biopsy, and due to the risk of tumor seeding, histological confirmation through surgery has been suggested, especially for lesions that are suspected to be gastrointestinal stromal tumors (GISTs) [4]. Although all GIST lesions have the potential for malignancy, controversies persist in regards to whether surgical morbidity or mortality is acceptable in the removal of SETs including lesions with low malignant potential $[1,5]$.

With the advancement of endoscopic skills and expertise, endoscopic submucosal dissection (ESD) has been performed in order to obtain tissue specimens, not only for lesions that are located in the submucosal (SM) layer, but even for those in the muscularis propria (MP) [6]. ESD allows for dissection below the tumor under direct vision [7]. Due to the potential of organ preservation and the less invasive nature of the ESD technique compared with surgical treatments, ESD might be preferred if therapeutic outcomes including safety indices are satisfied. The aim of this study was to evaluate the current evidence in regards to the therapeutic outcomes of ESD for the treatment of gastric SETs.

\section{METHODS}

\section{Literature search}

MEDLINE (through PubMed), EMBASE, and the Cochrane Central Register of Controlled Trials (CENTRAL) in the Cochrane Library were searched using common keywords related to ESD for the treatment of gastric SETs (from inception to July 2014). Medical Subject Headings (MeSH) terminology and user keywords that are commonly used in relevant articles were selected. The keywords included 'endoscopic submucosal dissection,' 'ESD,' 'endoscopic resection,' 'subepithelial tumors,' 'SET,' 'submucosal tumors,' and 'SMT' using Boolean operators. Only publications on human subjects were sought, and the bibliographies of relevant ar- ticles were also reviewed in order to identify additional studies.

\section{Selection criteria}

We included studies that met the following criteria: (1) the study was designed to evaluate the efficacy of ESD including endoscopic muscularis dissection for the treatment of gastric SETs and (2) the study included therapeutic outcomes (complete resection rate, recurrence rate or procedure-related adverse event rate) that enabled an evaluation of the feasibility of ESD for gastric SETs. The exclusion criteria were as follows: (1) studies with incomplete data, (2) review articles, (3) animal studies, or (4) letters or case articles.

\section{Selection of relevant studies}

Two of the authors (C.S.B. and G.H.B.) independently evaluated the eligibility of all studies that were retrieved from the databases. The evaluation was based on the predetermined selection criteria. The abstracts of all identified studies were reviewed to exclude irrelevant articles. Full-text reviews were performed to determine whether the inclusion criteria were satisfied by the remaining studies. Disagreements between the two evaluators were resolved by discussion or by consultation with a third author (D.J.K.).

\section{Assessment of methodological quality}

The methodological quality of the enrolled studies was assessed with the Newcastle-Ottawa scale. This tool comprises the following three parameters: the selection of the study population, the comparability of the groups, and the ascertainment of the exposure or outcome. Each parameter consists of subcategorized questions as follows: selection $(n=4)$, comparability $(n=1)$, and exposure or outcome $(n=3)[8,9]$. The stars awarded for each item allow for a rapid visual assessment of the methodological quality of the studies. A study can be awarded a maximum of nine stars, which indicates the highest quality. Two of the authors (C.S.B. and G.H.B.) independently evaluated the methodological quality of all the studies, and disagreements between the two evaluators were resolved by discussion or by consultation with a third author (D.J.K.). 


\section{Primary and modifier-based analyses}

Two of the authors (C.S.B. and G.H.B.) independently extracted the outcomes of all the studies, and disagreements between the two evaluators were resolved by discussion or by consultation with a third author (D.J.K.). The primary outcomes were as follows: (1) complete resection rate, which was the proportion of removed SETs with no components at the lateral or vertical margins on microscopic analysis; (2) recurrence rate, which was the proportion of SETs that reappeared at the site of the lesion (local recurrence) or synchronous, metachronous, or distant metastatic lesions; and (3) adverse event rate, which was the proportion of SETs whose treatment resulted in procedure-related gastric hemorrhage or perforation. We also performed sensitivity analyses based on the origin of the SETs in terms of the gastric layer and on the lesions that were confirmed to be GISTs. Both a cumulative analysis and a one-study-removed analysis were also performed.

\section{Statistics}

Comprehensive Meta-Analysis (CMA) software version 2.2.064 (Biostat; Borenstein M, Hedges L, Higgins J, and Rothstein H. Englewood, NJ, USA) was used for this meta-analysis. We calculated the pooled complete resection rate and the adverse event rate with $95 \%$ confidence intervals (CIs) from the enrolled studies. Heterogeneity was determined using the $I^{2}$ test, which was developed by Higgins; this test measures the percentage of total variation across studies [10]. $I^{2}$ was calculated with the following formula: $I^{2}(\%)=100 \times(\mathrm{Q}-\mathrm{df}) / \mathrm{Q}$, where $\mathrm{Q}$ is Cochrane's heterogeneity statistic and df signifies the degree of freedom. Negative values for $I^{2}$ were set to zero, and an $I^{2}$ value over 50\% was considered to be of substantial heterogeneity (range, o\% to 100\%) [11]. Pooled-effect sizes with $95 \%$ CIs were calculated with a random effects model and with the method of DerSimonian and Laird [12]. These results were confirmed by the $I^{2}$ test. A fixed effects model that included the inverse variance-weighted (Woolf's) method was used in the sensitivity analyses, including cumulative and one-study-removed analyses, based on the assumption of a common effect size shared by the studies within each subgroup [13,14]. Significance was set at $p=0.05$ in both models. Publication bias was evaluated with Begg's funnel plot, Egger's test of the intercept, Duval and Tweedie's trim and fill, and Begg and
Mazumdar's rank correlation test [15-19].

\section{RESULTS}

\section{Identification of relevant studies}

Fig. 1 contains a flow diagram that shows how relevant studies were identified. A total of 163 articles were identified by a search of three core databases. In all, 54 duplicate studies and an additional 92 studies were excluded during the initial screening through a review of the titles and abstracts. The full texts of the remaining 17 studies were then thoroughly reviewed. Among these studies, eight were excluded from the final analysis. The reasons for study exclusion during the final review were as follows: review article $(\mathrm{n}=2)$ or incomplete data ( $\mathrm{n}$ $=6$ ). The remaining nine studies were included in the final analysis [7,20-27].

\section{Characteristics of the studies}

Within the nine studies, we identified a total of 290 SETs from 288 patients (98 men and 190 women; 44 SETs originated from the SM layer and 246 SETs originated from the MP layer). The clinical characteristics of the patients from the included studies are shown in Tables 1 and 2. The included studies were published between 2006 and 2013. Seven studies were conducted in Asia [7,20,23-27] (three studies in China [24,25,27], two

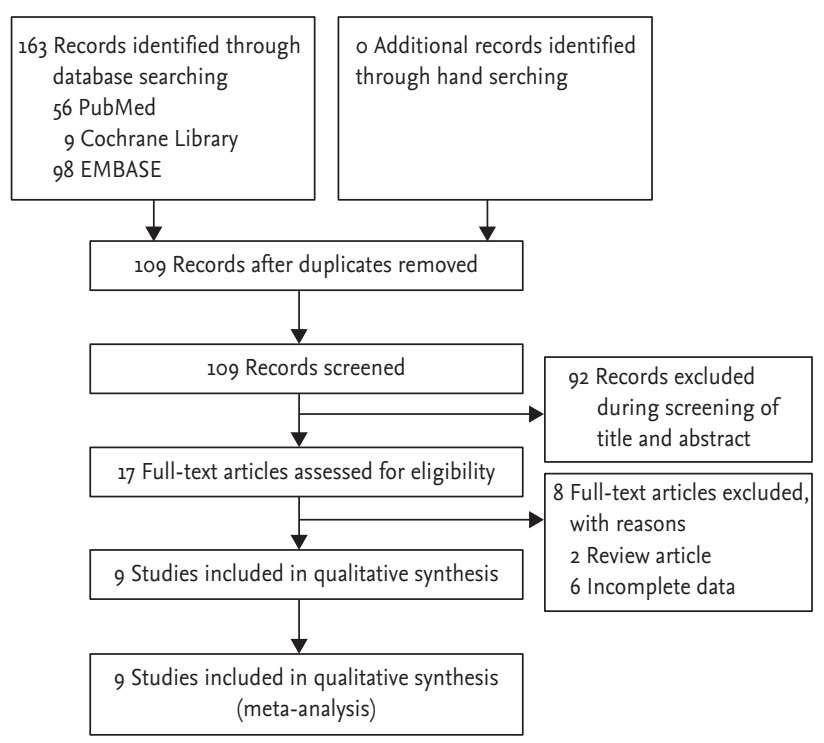

Figure 1. Flow diagram for identification of relevant studies. 


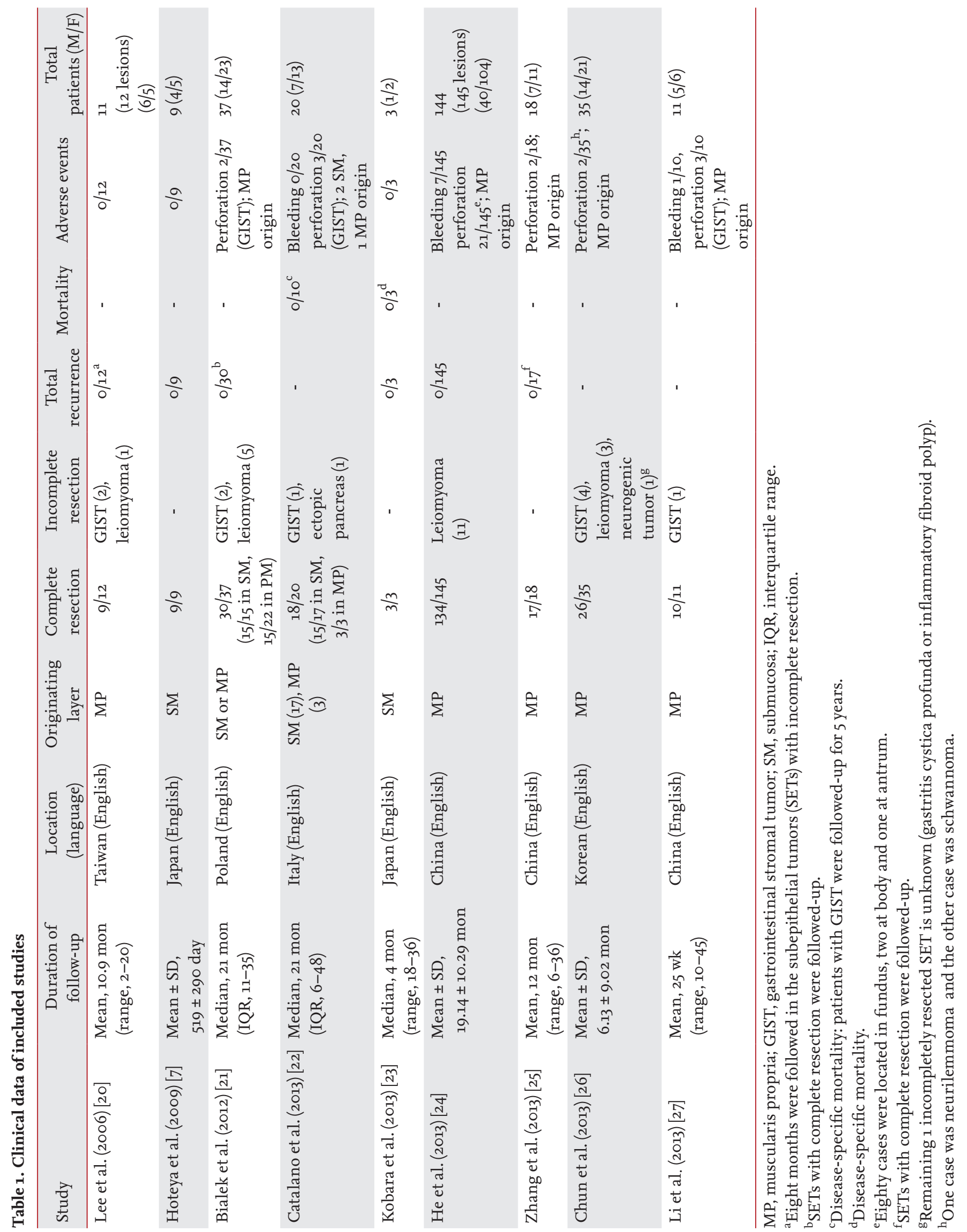




\section{KJIM ${ }^{-}$}

Study

Lee et al. (2006) [20]

Hoteya et al. (2009) [7]

Bialek et al. (2012) [21]

Catalano et al. (2013) [22]

Kobara et al. (2013) [23]

He et al. (2013) [24]

Zhang et al. (2013) [25]

Chun et al. (2013) [26]

Li et al. (2013) [27]

\begin{tabular}{ccc} 
& \multicolumn{2}{c}{ Statistice for each st } \\
Event rate & Lower limit & Upper limit \\
0.750 & 0.448 & 0.917 \\
0.950 & 0.525 & 0.997 \\
0.811 & 0.653 & 0.907 \\
0.900 & 0.676 & 0.975 \\
0.875 & 0.266 & 0.993 \\
0.924 & 0.868 & 0.957 \\
0.944 & 0.693 & 0.992 \\
0.734 & 0.575 & 0.860 \\
0.909 & 0.561 & 0.987 \\
0.862 & 0.789 & 0.913
\end{tabular}

Heterogeneity: $X^{2}=12.501, \mathrm{df}=8(p=0.130) ; I^{2}=36.006 \%$

Test for overall effect: $Z=6.929(p<0.001)$

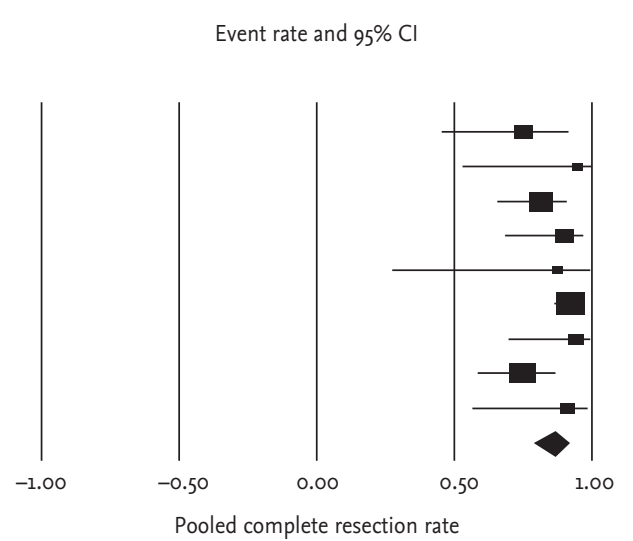

Event rate and $95 \% \mathrm{Cl}$

$p$ value

0.099

0.042

0.001

0.003

0.198

0.000

0.006

0.006

0.028

0.000

Figure 2. Total complete resection rate of enrolled studies. The size of each square is proportional to the study's weight. Diamond is the summary estimate from the pooled studies (random effect model). CI, confidence interval.

\begin{tabular}{lcccc} 
Study & \multicolumn{4}{c}{ Statistice for each study } \\
& Event rate & Lower limit & Upper limit & Z value \\
Lee et al. (2006) [20] & 0.038 & 0.002 & 0.403 & -2.232 \\
Hoteya et al. (2009) [7] & 0.050 & 0.003 & 0.475 & -2.029 \\
Bialek et al. (2012) [21] & 0.054 & 0.014 & 0.192 & -3.937 \\
Catalano et al. (2013) [22] & 0.150 & 0.049 & 0.376 & -2.770 \\
Kobara et al. (2013) [23] & 0.125 & 0.007 & 0.734 & -1.287 \\
He et al. (2013) [24] & 0.145 & 0.096 & 0.212 & -7.525 \\
Zhang et al. (2013) [25] & 0.111 & 0.028 & 0.352 & -2.773 \\
Chun et al. (2013) [26] & 0.057 & 0.014 & 0.202 & -3.850 \\
Li et al. (2013) [27] & 0.300 & 0.100 & 0.624 & -1.228 \\
& 0.130 & 0.094 & 0.176 & -10.353 \\
Heterogeneity: $X^{2}=7.377, \mathrm{df}=8(p=0.497) ; P^{2}=0 \%$ & & \\
Test for overall effect: Z $=-10.353(p<0.001)$ & & &
\end{tabular}

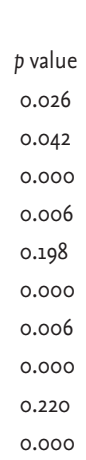

0.000

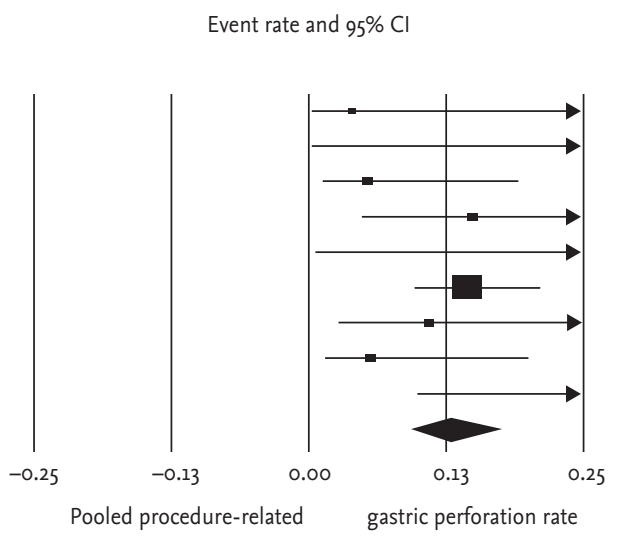

Figure 3. Total procedure-related adverse event rate of enrolled studies (gastric perforation). The size of each square is proportional to the study's weight. Diamond is the summary estimate from the pooled studies (random effect model). CI, confidence interval.

studies in Japan [7,23], one study in Korea [26], and one study in Taiwan [20]), and two studies were conducted in Europe (one study in Poland [21], and one study in Italy [22]). All of the included studies were written in English.

The age of the enrolled patients ranged from $52.3 \pm$ 18.2 to $65.3 \pm 6.3$ (mean $\pm \mathrm{SD}$ ). The diameter of the lesions ranged from $17.99 \pm 7.86$ to $38 \pm 22.1 \mathrm{~mm}$. The procedure time ranged from $32.29 \pm 20.55$, and the median procedure time was 119.1 minutes (range, 40 to 240 ). The procedure was performed under general anesthesia in 235 patients and under conscious sedation in 53 patients. Symptoms were present in 84 of the 216 identifiable patients. The symptoms were as follows: abdominal pain, discomfort, or heartburn $(n=65)$, gastrointestinal hemorrhage $(n=10)$, other symptoms $(n=7)$, or unidentifi- able symptoms $(\mathrm{n}=2)$. The follow-up duration after ESD ranged from a median of 4 months (range, 18 to 36 ) to 21 months (range, 6 to 48 ).

In terms of the methodological quality, the mean value of the awarded star was 4.56 (four stars [four studies] and 5 stars [five studies]) (Table 3). The enrolled studies shared relatively similar methodological quality, and thus, a sensitivity analysis based on the methodological quality was not performed.

\section{Overall efficacy and safety of ESD for the treatment of gastric SETs}

The overall efficacy of ESD for gastric SETs was evaluated using the complete resection rate, the recurrence rate, the survival rate, and the procedure-related adverse 


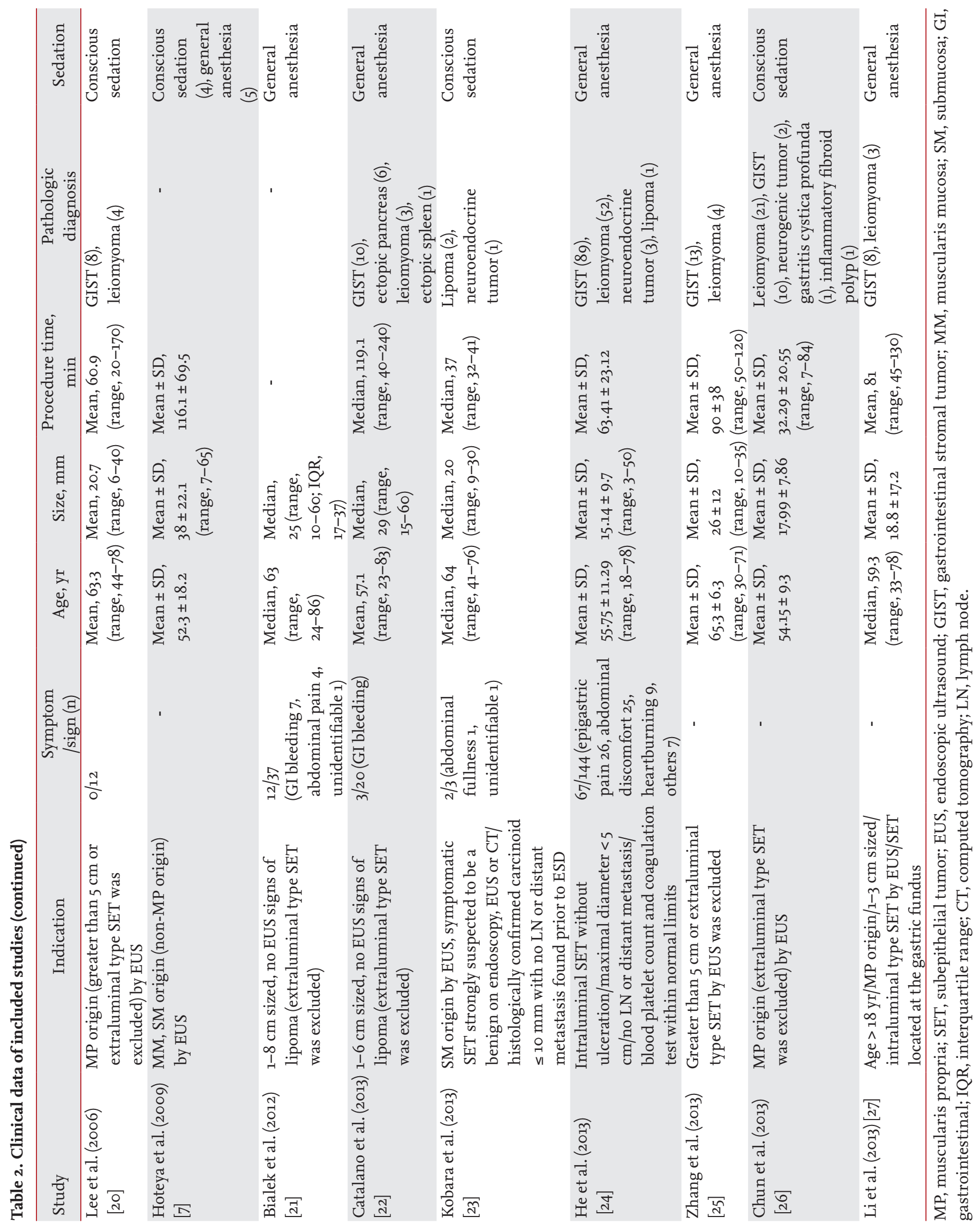


Table 3. Methodological quality of included studies measured by Newcastle-Ottawa scale

\begin{tabular}{|c|c|c|c|c|}
\hline Study & Selection & Comparability & Exposure or outcome & Total score \\
\hline Lee et al. (2006) [20] & 论论坛 & - & 访 & 4 \\
\hline Hoteya et al. (2009) [7] & 部动动 & - & 论败 & 5 \\
\hline Bialek et al. (2012) [21] & 论坛放 & - & $\hbar$ & 4 \\
\hline Catalano et al. (2013) [22] & 论认式 & - & 约 & 4 \\
\hline Kobara et al. (2013) [23] & 论该 & - & 论拧 & 4 \\
\hline He et al. (2013) [24] & 论论访 & - & 论拧 & 5 \\
\hline Zhang et al. (2013) [25] & 论地沶 & - & 论抎 & 5 \\
\hline Chun et al. (2013) [26] & 论放放 & - & 论㑔 & 5 \\
\hline Li et al. $(2013)[27]$ & 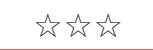 & - & 论柁 & 5 \\
\hline
\end{tabular}

event rate. The pooled complete resection rate was estimated to be $86.2 \%$ (95\% CI, 78.9 to $91.3 ; p<0.001$ ) (Fig. 2 ). The recurrence rate was not given in the enrolled studies, and no cases of disease or procedure-related mortality were reported. The pooled procedure-related gastric perforation rate was $13 \%$ (95\% CI, 9.4 to $17.6 ; p<0.001$ ) (Fig. 3). Among the 34 SETs that were incompletely resected, 20 cases were diagnosed as leiomyoma, 10 cases were GISTs, one case was an ectopic pancreas, one case was a neurogenic tumor, and two cases were unknown (Table 1).

\section{Sensitivity meta-analysis}

Sensitivity analyses were performed to show the robustness of the results of the main analysis. If the analysis was limited to the lesions that originated in the SM layer, the pooled complete resection rate was $91.4 \%$ (95\% CI, 77.9 to 97; $p<0.001$ ) (Fig. 4A). If the analysis was limited to the lesions that originated in the MP layer, the pooled complete resection rate was $84.4 \%$ (95\% CI, 78.7 to $88.8 ; p<0.001$ ) (Fig. $4 \mathrm{~B}$ ). If the analysis was limited to the lesions that were diagnosed as GISTs, the pooled complete resection rate was $82.7 \%$ (95\% CI, 70.5 to 90.6; p<0.001)(Fig. 4C). The pooled procedure-related gastric perforation rate of GISTs was $20.5 \%$ (95\% CI, 10.8 to 35.4; $p<0.001$ ) (Fig. 4D). Among the eight cases of gastric perforation that were associated with GISTs, six cases originated in the MP and two cases originated in the SM.

The cumulative meta-analysis of the enrolled studies in the order of year published showed a constant and slightly increasing trend in the complete resection rate and the procedure-related gastric perforation rate (Fig. 5). The one study-removed meta-analysis of the enrolled studies in the order of the year published showed consistent results (Fig. 6). The study by He et al. [24] demonstrated the most influential effect. This study has the largest enrolled population, and the methodological quality was relatively high among the enrolled studies.

\section{Publication bias}

A funnel plot for the included studies is illustrated in Fig. 7. These plots show a symmetrical shape.

In the publication bias analysis of the complete resection rate, Egger's regression test revealed that the intercept was 0.33 (95\% CI, -1.86 to $2.51 ; t$ value, $0.35 ; \mathrm{df}, 7 ; p=$ 0.37 [1-tailed] and $p=0.73$ [2-tailed]). A trim and fill analysis showed that two studies were missed or trimmed. The rank correlation test indicated a Kendall's tau of 0.08 with a continuity correction $(p=0.38$ [1-tailed] and $p=0.75$ [2-tailed]).

In the publication bias analysis of the procedure-related gastric perforation rate, Egger's regression test revealed that the intercept was -0.72 ( $95 \%$ CI, -1.99 to 0.55 ; $t$ value, 1.34; df, $7 ; p=0.11$ [1-tailed] and $p=0.22$ [2-tailed]). A trim and fill analysis demonstrated that two studies were missed or trimmed. The rank correlation test indicated a Kendall's tau of -0.14 with a continuity correction $(p=0.30$ [1-tailed] where $p=0.60$ [2-tailed]).

Overall, no evidence of publication bias was observed in this analysis.

\section{DISCUSSION}

According to the present study, ESD was found to be a technically feasible treatment modality for the treat- 


\begin{tabular}{lccccc} 
Study & \multicolumn{5}{c}{ Statistice for each study } \\
& Event rate & Lower limit & Upper limit & Z value & p value \\
Hoteya et al. (2009) [7] & 0.950 & 0.525 & 0.997 & 2.029 & 0.042 \\
Bialek et al. (2012) [21] & 0.969 & 0.650 & 0.998 & 2.390 & 0.017 \\
Catalano et al. (2013) [22] & 0.882 & 0.632 & 0.970 & 2.677 & 0.007 \\
Kobara et al. (2013) [23] & 0.875 & 0.266 & 0.993 & 1.287 & 0.198 \\
& 0.914 & 0.779 & 0.970 & 4.201 & 0.000
\end{tabular}

Heterogeneity: $X^{2}=1.006, \mathrm{df}=3(p=0.800) ; I^{2}=0 \%$

A

Test for overall effect: $Z=4.201(p<0.001)$
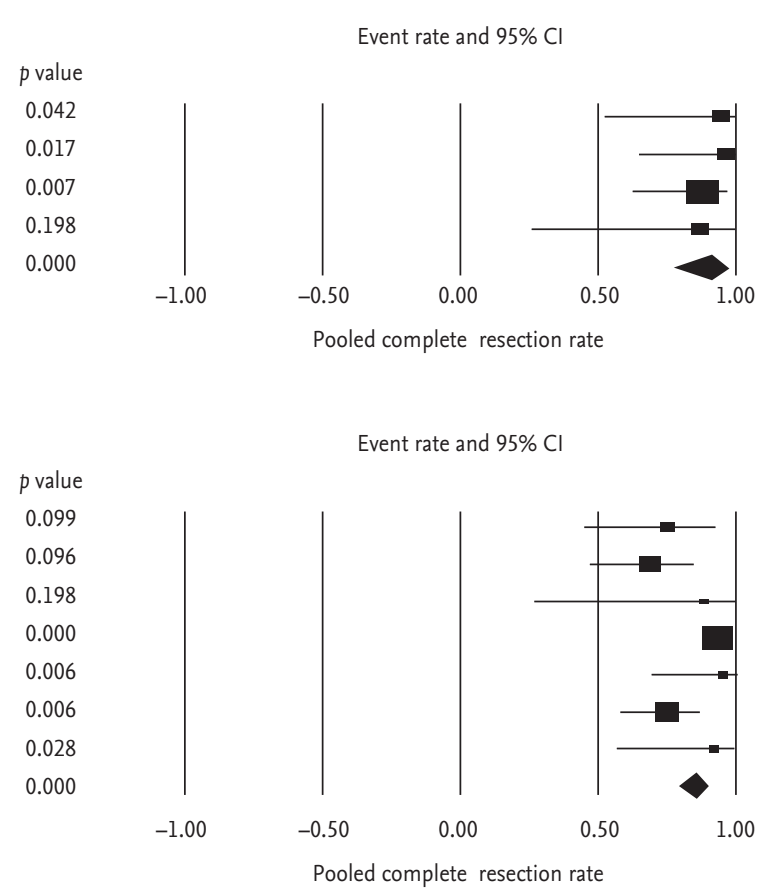

\begin{tabular}{lccccc} 
Study & \multicolumn{5}{c}{ Statistice for each study } \\
& Event rate & Lower limit & Upper limit & Z value & p value \\
Lee et al. (2006) [20] & 0.750 & 0.448 & 0.971 & 1.648 & 0.099 \\
Bialek et al. (2012) [21] & 0.682 & 0.466 & 0.840 & 1.665 & 0.096 \\
Catalano et al. (2013) [22] & 0.875 & 0.266 & 0.993 & 1.287 & 0.198 \\
He et al. (2013) [24] & 0.924 & 0.868 & 0.957 & 7.971 & 0.000 \\
Zhang et al. (2013) [25] & 0.944 & 0.693 & 0.992 & 2.753 & 0.006 \\
Chun et al. (2013) [26] & 0.743 & 0.575 & 0.860 & 2.743 & 0.006 \\
Li et al. (2013) [27] & 0.909 & 0.561 & 0.987 & 2.195 & 0.028 \\
& 0.844 & 0.787 & 0.888 & 8.648 & 0.000
\end{tabular}

$1 \%$
Test for overall effect: $Z=8.648(p<0.001)$
B

\begin{tabular}{lccccc} 
Study & \multicolumn{5}{c}{ Statistice for each study } \\
& Event rate & Lower limit & Upper limit & Z value & $p$ value \\
Lee et al. (2006) [20] & 0.750 & 0.377 & 0.937 & 1.346 & 0.178 \\
Bialek et al. (2012) [21] & 0.882 & 0.632 & 0.970 & 2.677 & 0.007 \\
Catalano et al. (2013) [22] & 0.900 & 0.533 & 0.986 & 2.084 & 0.037 \\
He et al. (2013) [24] & 0.994 & 0.917 & 1.000 & 3.658 & 0.000 \\
Chun et al. (2013) [26] & 0.600 & 0.297 & 0.842 & 0.628 & 0.530 \\
Li et al. (2013) [27] & 0.875 & 0.463 & 0.983 & 1.820 & 0.069 \\
& 0.827 & 0.705 & 0.906 & 4.414 & 0.000
\end{tabular}

C

Heterogeneity: $X^{2}=10.921, \mathrm{df}=5(p=0.053) ; P^{2}=54.218 \%$

Test for overall effect: $Z=4.414(p<0.001)$
Study

Lee et al. (2006) [20]
Bialek et al. (2012) [21]
Catalano et al. (2013) [22]
Chun et al. (2013) [26]
Li et al. (2013) [27]

\begin{tabular}{cccc} 
& \multicolumn{3}{c}{ Statistice for each study } \\
Event rate & Lower limit & Upper limit & Z value \\
0.056 & 0.003 & 0.505 & -1.947 \\
0.118 & 0.030 & 0.368 & -2.677 \\
0.300 & 0.100 & 0.624 & -1.228 \\
0.045 & 0.003 & 0.448 & -2.103 \\
0.375 & 0.125 & 0.715 & -0.699 \\
0.205 & 0.108 & 0.354 & -3.512
\end{tabular}

Heterogeneity: $X^{2}=5.040, \mathrm{df}=4(p=0.283) ; l^{2}=20.637 \%$

D Test for over all effect: $Z=-3.512(p<0.001)$
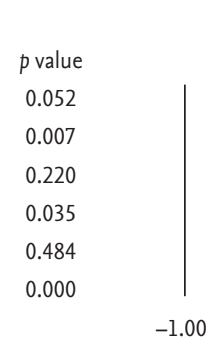

Event rate and $95 \% \mathrm{Cl}$

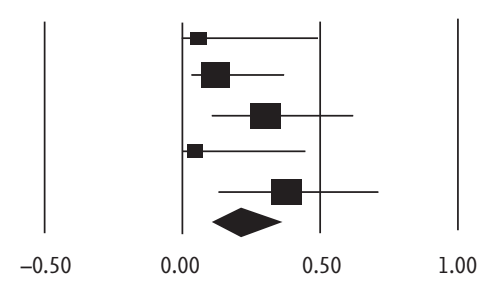

Pooled procedure-related gastric perforation rate

Figure 4. Sensitivity analysis. (A) Complete resection rate of lesions originated from the submucosal layer. (B) Complete resection rate of lesions originated from the muscularis propria layer. (C) Complete resection rate of gastrointestinal stromal tumor (GIST). (D) Total procedure-related gastric perforation rate of GIST. CI, confidence interval.

ment of SETs. The overall complete resection rate was $86.2 \%$, which increased to $91.4 \%$ for lesions that originated in the SM. However, for the lesions that originat- ed in the MP layer and for GISTs, the complete resection rate slightly decreased to $84.4 \%$ and $82.7 \%$, respectively. The procedure-related perforation rate was $13 \%$, which 
Study

$\begin{array}{lcccr} & \text { Point } & \text { Lower limit } & \text { Upper limit } & \text { Z value } \\ \text { Lee et al. (2006) [20] } & 0.750 & 0.448 & 0.917 & 1.648 \\ \text { Hoteya et al. (2009) [7] } & 0.805 & 0.558 & 0.931 & 2.345 \\ \text { Bialek et al. (2012) [21] } & 0.809 & 0.683 & 0.893 & 4.185 \\ \text { Catalano et al. (2013) [22] } & 0.829 & 0.724 & 0.899 & 5.036 \\ \text { Kobara et al. (2013) [23] } & 0.831 & 0.729 & 0.900 & 5.193 \\ \text { He et al. (2013) [24] } & 0.884 & 0.833 & 0.922 & 9.285 \\ \text { Zhang et al. (2013) [25] } & 0.888 & 0.839 & 0.923 & 9.655 \\ \text { Chun et al. (2013) [26] } & 0.862 & 0.812 & 0.900 & 9.774 \\ \text { Li et al. (2013) [27] } & 0.864 & 0.815 & 0.901 & 10.008 \\ & 0.864 & 0.815 & 0.901 & 10.008\end{array}$

A

Study
Lee et al. (2006) [20]
Hoteya et al. (2009) [7]
Bialek et al. (2012) [21]
Catalano et al. (2013) [22]
Kobara et al. (2013) [23]
He et al. (2013) [24]
Zhang et al. (2013) [25]
Chun et al. (2013) [26]
Li et al. (2013) [27]

\section{B}

\begin{tabular}{lccc} 
& \multicolumn{3}{c}{ Cumulative statistics } \\
Point & Lower limit & Upper limit & Z value \\
0.038 & 0.002 & 0.403 & -2.232 \\
0.044 & 0.006 & 0.254 & -3.014 \\
0.050 & 0.016 & 0.145 & -4.955 \\
0.086 & 0.039 & 0.179 & -5.503 \\
0.088 & 0.041 & 0.179 & -5.645 \\
0.129 & 0.090 & 0.181 & -9.333 \\
0.127 & 0.090 & 0.177 & -9.734 \\
0.121 & 0.086 & 0.166 & -10.403 \\
0.130 & 0.094 & 0.176 & -10.353 \\
0.130 & 0.094 & 0.176 & -10.353
\end{tabular}

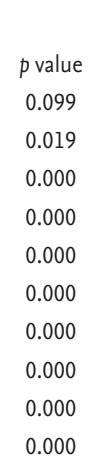

$p$ value

0.019

0.000

0.000

0.000

0.000

0.000

0.000

0.000

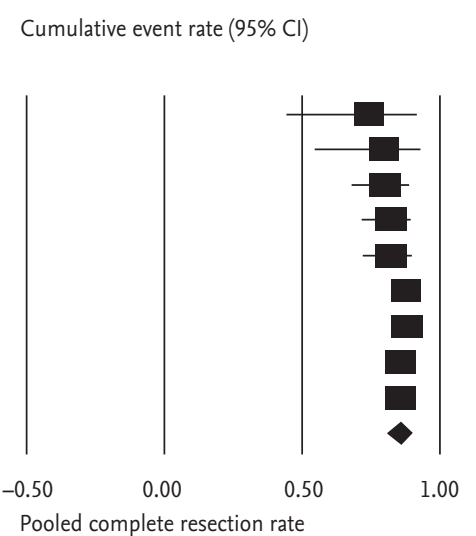

Cumulative event rate $(95 \% \mathrm{Cl})$

$$
\begin{gathered}
p \text { value } \\
0.026 \\
0.003 \\
0.000 \\
0.000 \\
0.000 \\
0.000 \\
0.000 \\
0.000 \\
0.000 \\
0.000
\end{gathered}
$$

Figure 5. Cumulative meta-analysis of enrolled studies. (A) For complete resection rate. (B) For procedure-related adverse event rate of enrolled studies (gastric perforation). CI, confidence interval.

increased to $20.5 \%$ for the lesions that were diagnosed as GISTs.

Therapeutic outcomes that were commonly shared among studies relevant to this topic were the complete resection rate, the recurrence rate, the survival rate and the procedure-related adverse event rate, especially the gastric perforation rate. However, the meaning of these indices is not fully understood with respect to the treatment of SETs. Complete resection was not based on the surgical specimens in order to confirm the completeness of the resection. Moreover, the follow-up duration was limited to confirm the recurrence or survival and whether the complete resection was associated with the prognosis [1]. Furthermore, we were unaware as to the natural history of the SETs, including GISTs, in our study $[2,28]$. The enrolled studies did not include an analysis divided by low-risk and high-risk GISTs. One of the main obstacles in the use of ESD for SETs is the deep location of these lesions. It is obvious that lesions in the MP layer are more likely to be perforated by ESD. However, the majority of the cases from the enrolled studies were managed by endoscopic clipping without additional surgical treatments. Thus, the procedure-related gastric perforation rate might also be less significant, although the low perforation rate should be maintained. To clarify the meaning of the therapeutic outcomes, large-scale, long-term studies are needed.

Another issue is the technique of ESD. Several variations of ESD for the removal of gastric SETs have been performed including the following: endoscopic muscularis dissection, endoscopic SM tunnel dissection, endoscopic enucleation, or endoscopic full-thickness resection with laparoscopic assistance, and laparoscopic and endoscopic cooperative surgery [29]. Endoscopic muscularis dissection is the procedure that is used for the removal of SETs that originate in the MP layer; in this method, the underlying proper muscle is dissected away from the tumor $[29,30]$. Thus, this method could 
Study

$\begin{array}{lcccr} & \text { Point } & \text { Lower limit } & \text { Upper limit } & \text { Z value } \\ \text { Lee et al. (2006) [20] } & 0.871 & 0.822 & 0.908 & 9.940 \\ \text { Hoteya et al. (2009) [7] } & 0.862 & 0.812 & 0.900 & 9.830 \\ \text { Bialek et al. (2012) [21] } & 0.875 & 0.823 & 0.912 & 9.446 \\ \text { Catalano et al. (2013) [22] } & 0.861 & 0.810 & 0.900 & 9.576 \\ \text { Kobara et al. (2013) [23] } & 0.864 & 0.815 & 0.901 & 9.925 \\ \text { He et al. (2013) [24] } & 0.818 & 0.742 & 0.875 & 6.576 \\ \text { Zhang et al. (2013) [25] } & 0.860 & 0.810 & 0.899 & 9.671 \\ \text { Chun et al. (2013) [26] } & 0.889 & 0.841 & 0.924 & 9.899 \\ \text { Li et al. (2013) [27] } & 0.862 & 0.812 & 0.900 & 9.774 \\ & 0.864 & 0.815 & 0.901 & 10.008\end{array}$

A
Study

Lee et al. (2006) [20]

Hoteya et al. (2009) [7]

Bialek et al. (2012) [21]

Catalano et al. (2013) [22]

Kobara et al. (2013) [23]

He et al. (2013) [24]

Zhang et al. (2013) [25]

Chun et al. (2013) [26]

Li et al. (2013) [27]

\begin{tabular}{lccc} 
& \multicolumn{3}{c}{ Statistics with study removed } \\
Point & Lower limit & Upper limit & Z value \\
0.132 & 0.096 & 0.180 & -10.152 \\
0.132 & 0.095 & 0.179 & -10.178 \\
0.137 & 0.099 & 0.188 & -9.672 \\
0.128 & 0.091 & 0.176 & -9.980 \\
0.130 & 0.094 & 0.176 & -10.273 \\
0.109 & 0.064 & 0.178 & -7.164 \\
0.131 & 0.094 & 0.179 & -9.978 \\
0.137 & 0.098 & 0.187 & -9.696 \\
0.121 & 0.086 & 0.166 & -10.403 \\
0.130 & 0.094 & 0.176 & -10.353
\end{tabular}

B

$$
\begin{gathered}
p \text { value } \\
0.000 \\
0.000 \\
0.000 \\
0.000 \\
0.000 \\
0.000 \\
0.000 \\
0.000 \\
0.000 \\
0.000
\end{gathered}
$$

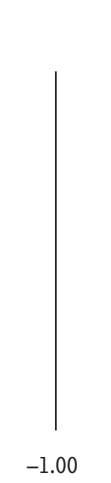

Event rate $(95 \% \mathrm{Cl})$ with study removed

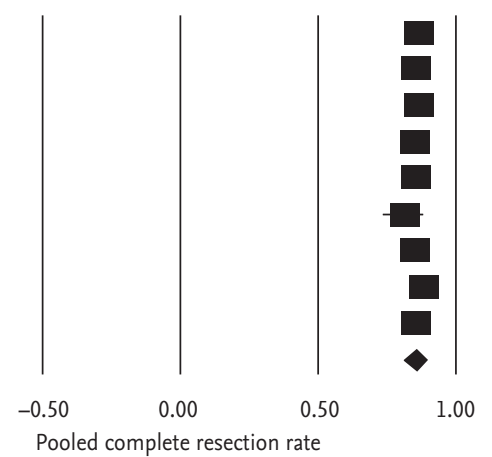

Event rate $(95 \% \mathrm{Cl})$ with study removed

$$
\begin{gathered}
p \text { value } \\
0.000 \\
0.000 \\
0.000 \\
0.000 \\
0.000 \\
0.000 \\
0.000 \\
0.000 \\
0.000 \\
0.000
\end{gathered}
$$

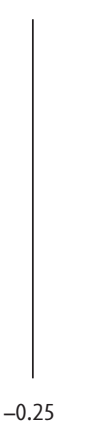

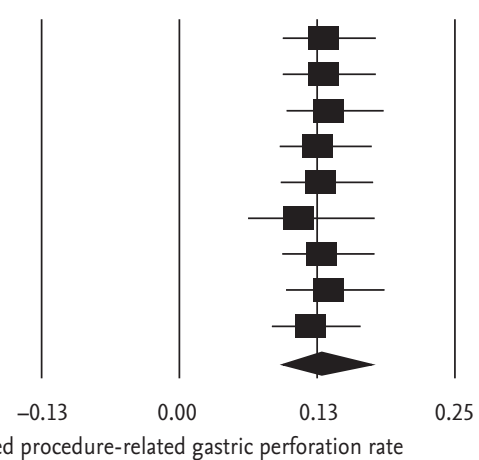

Figure 6. One study removed meta-analysis of enrolled studies. (A) For complete resection rate. (B) For procedure-related adverse event rate of enrolled studies (gastric perforation). CI, confidence interval.

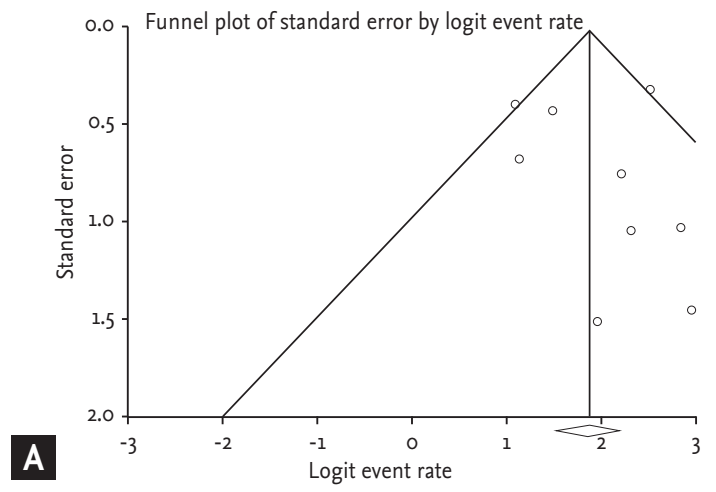

\section{B}

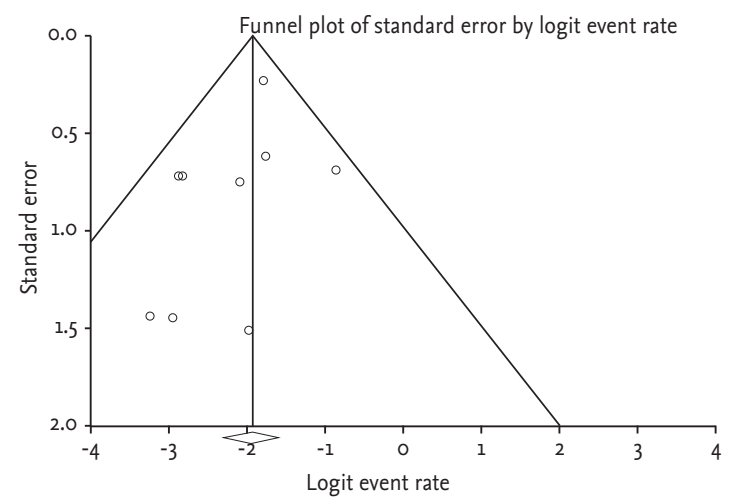

Figure 7. Funnel plot for publication bias. (A) Complete resection rate. (B) Funnel plot for publication bias (procedure-related adverse event rate; gastric perforation).

be distinguished from traditional ESD. However, in this meta-analysis, none of the enrolled studies differentiated these procedures, but rather, the traditional ESD technique was applied directly for the removal of SETs that originated in the MP. Therefore, therapeutic out- comes of ESD including endoscopic muscularis dissection were combined, and sensitivity analyses were performed and divided by the SET according to the layer of origin. Although this meta-analysis included only the ESD procedure, the various techniques described above 
are a candidate to be the mainstay of future treatment modalities.

Despite the technical feasibility of ESD for the treatment of gastric SETs, as described above, the most important issue is the establishment of indications. The selection criteria for the patients were similar among the enrolled studies, although a size discrepancy was noted among the indications (Table 2). Only EUS was performed to determine the indications for the patients in all of the enrolled studies. Additionally, the layer in which the tumors originated, whether the tumors were of the extraluminal or intraluminal type, and the findings that indicated benign lesions such as lipoma or malignant lesions such as GIST were described. However, considering that in one of the enrolled studies, EUS was only accurate in $73 \%$ of the lesions for the determination of the layer of tumor origin, another diagnostic modality or factor should be added for the determination of the indications [21]. Extraluminal type SETs and large-sized lesions were commonly excluded because of the technical difficulties and the high perforation risk. However, other factors such as a wide base or a narrow base, whether the lesions were well-demarcated or not, and whether endoscopic or EUS findings predicted the adhesion severity were not included in the indications.

This study is the first meta-analysis on the efficacy of ESD for the treatment of SETs. The strength of this study is the rigorous literature search. When possible, potential modifiers were detected within the articles, and sensitivity analyses were performed to confirm the robustness of the results. Despite the strengths, there are also several limitations. First, the number of enrolled patients in each study was too small. Each study has a potential of selection bias, and thus, the enrolled population might be heterogeneous. Second, the follow-up duration was too short. With the data from this meta-analysis, it is impossible to predict the long-term efficacy or the prognostic indices. Third, the experience and skill of the endoscopists with respect to ESD were not considered. ESD, including endoscopic muscularis dissection, is a highly complicated procedure that requires a high level of skill. The therapeutic outcomes including the procedure-related adverse event rates might be dependent on the experience of endoscopists. The limitations described above could also be a cause of heterogeneity and bias. Large-scale, well-organized, long- term follow-up studies are therefore needed to establish proper indications of ESD for the treatment of SETs.

In conclusion, ESD including endoscopic muscularis dissection is a technically feasible procedure for the diagnosis and treatment of SETs. For the development of proper indications for ESD for the treatment of SETs, additional studies are needed.

\section{KEY MESSAGE}

1. Current evidence indicates endoscopic submucosal dissection (ESD) including endoscopic muscularis dissection is technically feasible procedure for the treatment of subepithelial tumors (SETs).

2. However, selection bias is suspected from the enrolled studies. For the development of proper indication about ESD for SETs, further studies are needed.

\section{Conflict of interest}

No potential conflict of interest relevant to this article was reported.

\section{REFERENCES}

1. Hwang JH, Rulyak SD, Kimmey MB; American Gastroenterological Association Institute. American Gastroenterological Association Institute technical review on the management of gastric subepithelial masses. Gastroenterology 2006;130:2217-2228.

2. Lim YJ, Son HJ, Lee JS, et al. Clinical course of subepithelial lesions detected on upper gastrointestinal endoscopy. World J Gastroenterol 2010;16:439-444.

3. Kojima T, Takahashi H, Parra-Blanco A, Kohsen K, Fujita R. Diagnosis of submucosal tumor of the upper GI tract by endoscopic resection. Gastrointest Endosc 1999;50:516522.

4. Blay JY, Bonvalot S, Casali P, et al. Consensus meeting for the management of gastrointestinal stromal tumors: report of the GIST Consensus Conference of 2021 March 2004, under the auspices of ESMO. Ann Oncol 2005;16:566-578.

5. Fletcher CD, Berman JJ, Corless C, et al. Diagnosis of gastrointestinal stromal tumors: a consensus approach. Int J 
Surg Pathol 2002;10:81-89.

6. Goto O, Uraoka T, Horii J, Yahagi N. Expanding indications for ESD: submucosal disease (SMT/carcinoid tumors). Gastrointest Endosc Clin N Am 2014;24:169-181.

7. Hoteya S, lizuka T, Kikuchi D, Yahagi N. Endoscopic submucosal dissection for gastric submucosal tumor, endoscopic sub-tumoral dissection. Dig Endosc 2009;21:266269.

8. Deeks JJ, Dinnes J, D’Amico R, et al. Evaluating non-randomised intervention studies. Health Technol Assess 2003;7:1-173.

9. Stang A. Critical evaluation of the Newcastle-Ottawa scale for the assessment of the quality of nonrandomized studies in meta-analyses. Eur J Epidemiol 2010;25:603-605.

10. Higgins JP, Thompson SG. Quantifying heterogeneity in a meta-analysis. Stat Med 2002;21:1539-1558.

11. Higgins JP, Thompson SG, Deeks JJ, Altman DG. Measuring inconsistency in meta-analyses. BMJ 2003;327:557-560.

12. DerSimonian R, Laird N. Meta-analysis in clinical trials. Control Clin Trials 1986;7:177-188.

13. Borenstein M, Hedges LV, Higgins JP, Rothstein HR. Fixed-effect versus random-effects models. In: Borenstein M, Hedges LV, Higgins JP, Rothstein HR, eds. Introduction to Meta-Analysis. Chichester: John Wiley \& Sons, 2009:59-102.

14. Hedges LV, Olkin I. Statistical Methods for Meta-Analysis. Orlando: Academic Press, 1985.

15. Duval S, Tweedie R. Trim and fill: a simple funnel-plotbased method of testing and adjusting for publication bias in meta-analysis. Biometrics 2000;56:455-463.

16. Sutton AJ, Abrams KR, Jones DR, Sheldon TA, Song F. Methods for Meta-Analysis in Medical Research. Chichester: Wiley, 2000.

17. Sterne JA, Egger M. Funnel plots for detecting bias in meta-analysis: guidelines on choice of axis. J Clin Epidemiol 2001;54:1046-1055.

18. Begg CB, Mazumdar M. Operating characteristics of a rank correlation test for publication bias. Biometrics 1994;50:1088-1101.

19. Egger M, Davey Smith G, Schneider M, Minder C. Bias in meta-analysis detected by a simple, graphical test. BMJ 1997;315:629-634.

20. Lee IL, Lin PY, Tung SY, Shen CH, Wei KL, Wu CS. Endoscopic submucosal dissection for the treatment of intraluminal gastric subepithelial tumors originating from the muscularis propria layer. Endoscopy 2006;38:10241028.

21. Bialek A, Wiechowska-Kozlowska A, Pertkiewicz J, et al. Endoscopic submucosal dissection for treatment of gastric subepithelial tumors (with video). Gastrointest Endosc 2012;75:276-286.

22. Catalano F, Rodella L, Lombardo F, et al. Endoscopic submucosal dissection in the treatment of gastric submucosal tumors: results from a retrospective cohort study. Gastric Cancer 2013;16:563-570.

23. Kobara H, Mori H, Rafiq K, et al. Indications of endoscopic submucosal dissection for symptomatic benign gastrointestinal subepithelial or carcinoid tumors originating in the submucosa. Mol Clin Oncol 2013;1:10021008.

24. He Z, Sun C, Wang J, et al. Efficacy and safety of endoscopic submucosal dissection in treating gastric subepithelial tumors originating in the muscularis propria layer: a single-center study of 144 cases. Scand J Gastroenterol 2013;48:1466-1473.

25. Zhang S, Chao GQ, Li M, Ni GB, Lv B. Endoscopic submucosal dissection for treatment of gastric submucosal tumors originating from the muscularis propria layer. Dig Dis Sci 2013;58:1710-1716.

26. Chun SY, Kim KO, Park DS, et al. Endoscopic submucosal dissection as a treatment for gastric subepithelial tumors that originate from the muscularis propria layer: a preliminary analysis of appropriate indications. Surg Endosc 2013;27:3271-3279.

27. Li L, Wang F, Wu B, Wang Q, Wang C, Liu J. Endoscopic submucosal dissection of gastric fundus subepithelial tumors originating from the muscularis propria. Exp Ther Med 2013;6:391-395.

28. Kim MY, Jung HY, Choi KD, et al. Natural history of asymptomatic small gastric subepithelial tumors. J Clin Gastroenterol 2011;45:330-336.

29. Abe N, Takeuchi H, Ooki A, et al. Recent developments in gastric endoscopic submucosal dissection: towards the era of endoscopic resection of layers deeper than the submucosa. Dig Endosc 2013;25 Suppl 1:64-70.

30. Liu BR, Song JT, Qu B, Wen JF, Yin JB, Liu W. Endoscopic muscularis dissection for upper gastrointestinal subepithelial tumors originating from the muscularis propria. Surg Endosc 2012;26:3141-3148. 


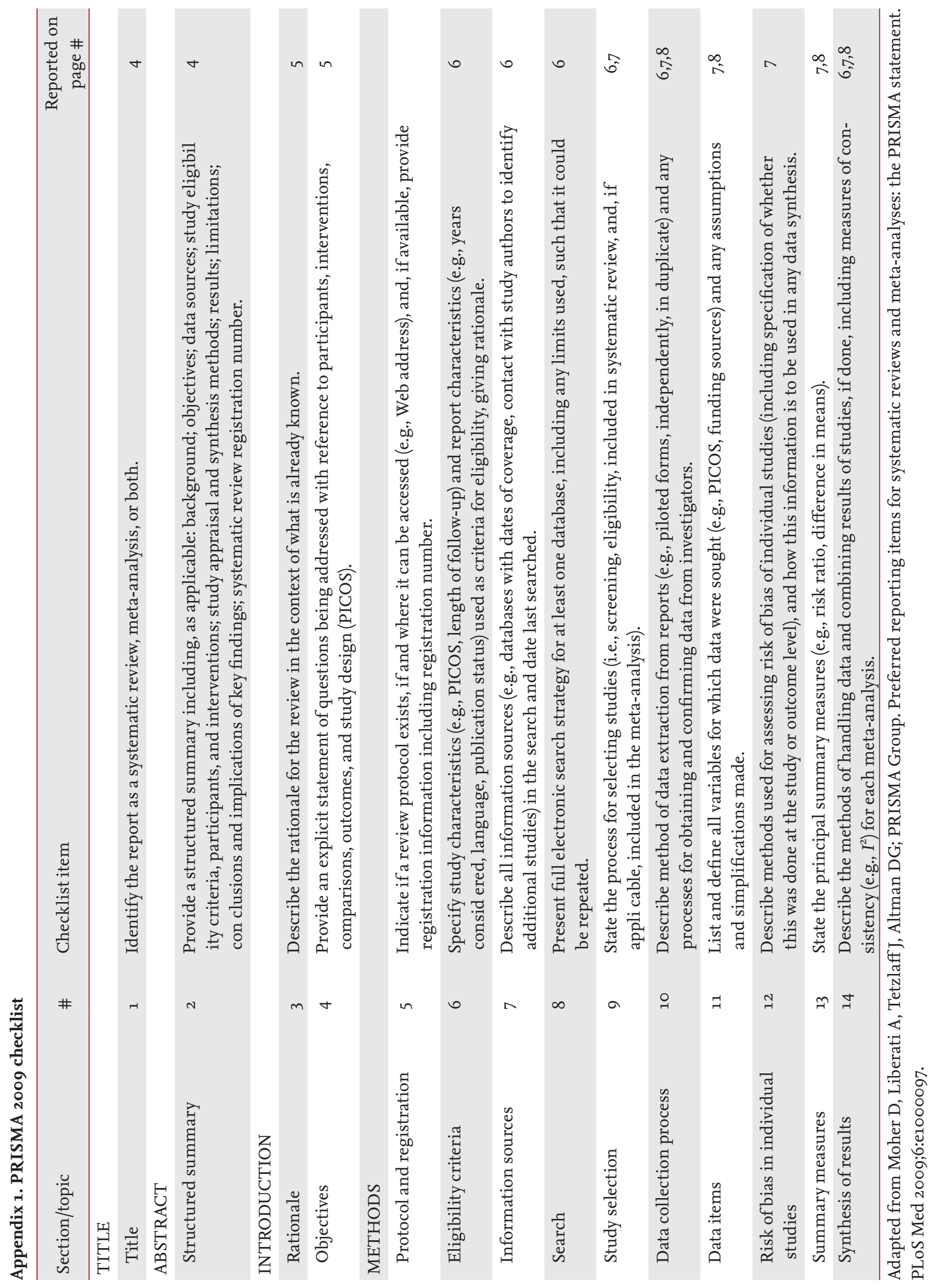

